\title{
Reducing Inhomogeneity Artifacts in Functional MRI of Human Brain Activation-Thin Sections vs Gradient Compensation
}

\author{
Klaus-Dietmar Merboldt, Jürgen Finsterbusch, and Jens Frahm ${ }^{1}$ \\ Biomedizinische NMR Forschungs GmbH am Max-Planck-Institut für biophysikalische Chemie, D-37070 Göttingen, Germany
}

Received August 18, 1999; revised March 24, 2000

\begin{abstract}
We evaluated two methods for correcting inhomogeneity-induced signal losses in magnetic resonance gradient-echo imaging that either use gradient compensation or simply acquire thin sections. The strategies were tested in the human brain in terms of achievable quality of T2*-weighted images at the level of the hippocampus and of functional activation maps of the visual cortex. Experiments were performed at $2.0 \mathrm{~T}$ and based on singleshot echo-planar imaging at $2.0 \times 2.0 \mathrm{~mm}^{2}$ resolution, $4 \mathrm{~mm}$ section thickness, and $2.0 \mathrm{~s}$ temporal resolution. Gradient compensation involved a sequential 16-step variation of the refocusing lobe of the slice-selection gradient (TR/TE $=125 / 53 \mathrm{~ms}$, flip angle $15^{\circ}$ ), whereas thin sections divided the 4-mm target plane into either four $1-\mathrm{mm}$ or eight $0.5-\mathrm{mm}$ interleaved multislice acquisitions (TR/TE $=2000 / 54 \mathrm{~ms}$, flip angle $70^{\circ}$ ). B oth approaches were capable of alleviating the inhomogeneity problem for structures in the base of the brain. When compared to standard 4-mm EPI, functional mapping in the visual cortex was partially compromised because of a lower signal-to-noise ratio of inhomogeneitycorrected images by either method. Relative to each other, consistently better results were obtained with the use of contiguous thin sections, in particular for a thickness of $1 \mathrm{~mm}$. Multislice acquisitions of thin sections require minimal technical adjustments. (c) 2000 A cademic Press
\end{abstract}

Key Words: functional magnetic resonance imaging; hippocampus; human brain activation; susceptibility artifacts; visual stimulation.

\section{INTRODUCTION}

Gradient-echo magnetic resonance imaging (MRI) sequences such as FLASH and EPI rely on gradient-recalled magnetizations that are sensitive to off-resonance effects and therefore cause intravoxel signal dephasing and/or geometric distortions in the presence of magnetic field inhomogeneities. Whereas problems due to macroscopic structure-induced susceptibility gradients are usually minimized by reducing the echo time and voxel size in anatomic MRI, they are particularly emphasized in functional mapping of human brain activation. Pertinent applications commonly employ low spatial resolution to maximize volume coverage per repetition time

\footnotetext{
${ }^{1}$ To whom correspondence should be addressed at Biomedizinische NMR Forschungs GmbH, D-37070 Göttingen, Germany. E-mail: jfrahm@gwdg.de.
}

(TR) and, even more importantly, require the use of prolonged gradient echo times (TE) to enhance the sensitivity to microscopic tissue-specific susceptibility gradients. In gray matter, these latter primarily reflect differences in the concentration of paramagnetic deoxyhemoglobin as the underlying contrast parameter for blood oxygenation level-dependent (BOLD) MRI of brain function.

So far, the majority of brain activation studies has focused on investigations of primary systems such as visual, sensorimotor, and auditory cortices with only small to mild affection by structural inhomogeneities. However, with increasing interest in higher order cognitive and mental processing, more attention is drawn to areas such as the hippocampus and parahippocampal gyrus which are involved in memory and learning as well as in various psychiatric and neurodegenerative disorders. Because these areas are located in the vicinity of air-filled spaces of the nasal cavity and auditory canal, the corresponding magnetic susceptibility differences at air-tissue or bone-tissue interfaces result in severe distortions and regional signal losses in long-TE gradient-echo images.

Proposals for overcoming the inhomogeneity problem and, more recently, for developing suitable strategies for BOLD activation studies include (i) a reduction of pixel size and slice thickness (1-4), (ii) radiofrequency (RF) profiling (5-7), (iii) compensation of the slice-selection gradient $(8-17)$, or (iv) combinations thereof; e.g., see (18). The underlying assumption is that these strategies recover signals lost by macroscopic magnetic field inhomogeneities but retain the BOLD MRI contrast, i.e., the sensitivity to microscopic deoxyhemoglobinrelated susceptibility differences. Thus, before attempting to functionally map structures in the human hippocampal formation, it seems mandatory to compare the performance of the correction strategies in terms of achievable image quality and, independently, to test their ability to map brain activation in the absence of inhomogeneities.

Accordingly, the purpose of this work was twofold. In a first part we assessed the efficiency of the gradient compensation and thin section methods to correct for anatomic MRI artifacts in images of the base of the brain and to recover signals close to air-tissue boundaries. Technically, the gradient compensation method involves sequential acquisitions of the same sec- 
tion but with different refocusing strengths of the slice-selection gradient. Compensated maps are then obtained from a suitable subset of images either by maximum intensity projection (MIP) or after Fourier transformation (FT). The thin section method comprises a simple multislice acquisition of very thin sections and a subsequent summation defining the target plane, here $4 \mathrm{~mm}$. In either case, MRI acquisitions are based on gradient-echo EPI sequences with identical temporal $(2.0 \mathrm{~s})$ and spatial resolution $\left(2.0 \times 2.0 \mathrm{~mm}^{2}\right)$.

In a second part we evaluated whether the two correction schemes affect the functional recording of brain activation in an area uncompromised by macroscopic susceptibility differences. Activation of the visual cortex was elicited with the use of a robust checkerboard paradigm leading to well-established spatiotemporal response profiles (19). This also excludes potential confounds in this methodologic baseline study that may arise from the subjects' differences in performing a memory task or the not yet fully investigated hemodynamic responsiveness of hippocampal areas.

\section{METHODS}

Apart from a large number of subjects contributing to pilot experiments, six healthy adults (mean age $26.8 \pm 3.3$ years) participated in the final study protocols. Written informed consent was obtained before all examinations. Experiments were performed at 2.0 T (Siemens Vision, Erlangen, Germany) with use of the standard head coil and gradients (maximum amplitude $25 \mathrm{mT} \mathrm{m}^{-1}$ ).

Dynamic BOLD MRI was based on a single-shot blipped gradient-echo EPI sequence (symmetric coverage of $k$ space, $1470 \mathrm{~Hz}$ bandwidth per pixel). The duration of the sliceselective RF pulse (Hanning-filtered sinc profile with one side lobe) was $3.84 \mathrm{~ms}$ (mean $\mathrm{TE}=53 \mathrm{~ms}$, minimum section thickness of $1 \mathrm{~mm}$ ) for gradient compensation and $5.34 \mathrm{~ms}$ ( $\mathrm{TE}=54 \mathrm{~ms}$, minimum section thickness $0.5 \mathrm{~mm}$ ) for the thin section method. The resulting section profiles were experimentally verified to allow for interleaved multislice imaging of contiguous sections with signal reductions of less than $10 \%$ relative to single sections. T2*-weighted images were acquired with a flip angle of $70^{\circ}$ for TR $=2000 \mathrm{~ms}$ and a flip angle of $15^{\circ}$ for $\mathrm{TR}=125 \mathrm{~ms}$ to minimize the influence of T1 saturation and inflow effects. An effective temporal resolution of $2 \mathrm{~s}$ was chosen in line with the conditions of typical neuroimaging studies. In all cases the spatial resolution was $2.0 \times 2.0 \mathrm{~mm}^{2}$ using a data matrix of $112 \times 128$ together with a $\frac{7}{8}$ rectangular field-of-view covering $224 \times 256 \mathrm{~mm}^{2}$. For display purposes the images were zoomed without smoothing. Oblique sections were oriented along the long axis of the hippocampus or followed the calcarine fissure for mapping the visual cortex.

\section{Strategies for Inhomogeneity Correction}

Compensated images and corresponding functional maps were compared to acquisitions without inhomogeneity correc- tion but with otherwise identical measurement parameters. The thin section method involved interleaved multislice acquisitions of four or eight contiguous sections with thicknesses of 1 or $0.5 \mathrm{~mm}$, respectively, that subdivided the 4-mm target plane $(\mathrm{TR}=2000 \mathrm{~ms})$. For gradient compensation the refocusing part of the slice-selection gradient for the 4-mm section was incremented in 16 steps to avoid severe truncation artifacts after FT and, more importantly, to account for a wide range of unknown and spatially heterogenous susceptibility offsets. The use of a fewer number of gradient steps would be possible only with prior knowledge about susceptibility-induced errors and not result in a generally applicable and user-independent compensation method.

To achieve a temporal resolution of $2.0 \mathrm{~s}$, the 16 images of the same section were acquired sequentially with $\mathrm{TR}=125$ ms. The offsets for the refocusing lobe of the slice-selection gradient varied by about $\pm 100 \%$, i.e., $\pm 5.0 \mathrm{mT} \mathrm{m}^{-1}$ for a rephasing lobe of $1.8 \mathrm{~ms}$ duration. These values were found to completely span the range necessary to recover a maximum of lost signal in images of the base of the brain. It was also used for visual activation studies to provide realistic conditions for future functional applications to the hippocampus.

Data processing of the MRI system was modified to allow for an automated online calculation of inhomogeneitycorrected images. For the thin section method this referred to a simple summation of 4 or 8 magnitude images from the corresponding subsections of the 4-mm target plane. Gradient-compensated maps were reconstructed either by a pixelwise MIP of the 16 images with incremented refocusing gradients or by taking magnitude images after a complex FT.

\section{Visual Activation Studies}

The activation protocol consisted of 6 repetitive cycles of $20 \mathrm{~s}$ of checkerboard stimulation $(5 \mathrm{~Hz})$ and $20 \mathrm{~s}$ of gray light (same mean luminescence). Baseline conditions were established by an initial 28 -s period of gray light discarding images from the first $8 \mathrm{~s}$ for equilibration purposes. The computergenerated stimuli were projected into the magnet by a setup covering $40^{\circ} \times 30^{\circ}$ of the subject's visual field (Schäfter \& Kirchhoff, Hamburg, Germany). Functional mapping was based on quantitative maps of correlation coefficients obtained by a pixel-by-pixel cross-correlation (20) of signal intensity time courses with a suitable reference function (4 s delay relative to stimulus onset) and a statistical analysis exploiting the individual noise distribution (21). The approach utilizes a $99.99 \%$ percentile rank threshold for the identification of highly activated foci corresponding to a selection criterion of $p<0.01$ and iteratively integrates directly neighboring pixels above a $95 \%$ threshold for area delineation. Thresholded correlation coefficient maps were color-coded and superimposed onto mean EPI raw images from the same series. This avoids ambiguities caused by geometric distortions of EPI-derived 
activation maps relative to anatomic gradient-echo or spinecho images. The activation paradigm and the resulting spatiotemporal brain responses have been previously described (19).

\section{RESULTS AND DISCUSSION}

\section{T2*-Weighted Imaging of the Hippocampal Formation}

Figure 1a demonstrates susceptibility-induced image artifacts that are typical for T2*-weighted BOLD MRI of the human hippocampus. As shown in Fig. 1b for the three selected regions-of-interest (ROI) the gradient-echo EPI signal intensity losses in the vicinity of the nasal cavity and auditory canal may be partially recovered by recording images with differently compensated slice refocusing gradients. Obviously, different regions exhibit susceptibility-induced field gradients with different amplitudes and signs. For example, in comparison with data from the occipital cortex, the inhomogeneity offset in the nasal tract region is opposite to that in the vicinity of the auditory tract. As shown in Fig. 1c FT along the direction of the slice refocusing gradient collapses the spread of variable compensations along the resulting image axis. It turns out that the experimental data both without and with FT yield a certain broadness, so that for practical purposes compensated maps were obtained either by a pixelwise MIP and averaging of the three maximum intensities or-after FT — by averaging of the three central images. Pilot experiments demonstrated that MIP maps based on only a single maximum intensity value or FT maps representing only the central FT image performed poorer in terms of signal recovery compared with the present approaches. Combinations of more than three images resulted in reduced signal-to-noise (SNR) because of the incorporation of very low intensity images.

The image quality achieved for the various inhomogeneity correction schemes is illustrated in Fig. 2 for the same subject as in Fig. 1. In addition to an anatomic flow-sensitized gradient-echo acquisition (top left) and an uncorrected $\mathrm{T} 2 *_{\text {- }}$ weighted image (top right), the figure comprises a gradientcompensated MIP map (middle left) and a corresponding FT map (middle right) as well as the results of two multislice acquisitions summing up either four 1-mm (bottom left) or eight 0.5 -mm-thick sections (bottom right). Although gradient compensation with both the MIP and the FT variant results in significant recovery of lost signal, the strategy does not reach the slightly better performance of the simple thin section method. This finding was consistent with all subjects. In fact, the use of a 1-mm section seems to be sufficient to substantially reduce susceptibility-induced artifacts at $2.0 \times 2.0 \mathrm{~mm}^{2}$ inplane resolution. This is also documented by the good quality of the individual 1-mm images (not shown). No further improvements are discernible when reducing the section thickness to $0.5 \mathrm{~mm}$. It should also be noted that $2-\mathrm{mm}$ sections were not able to sufficiently recover dephased signals.

A closer inspection of the inhomogeneity-corrected images
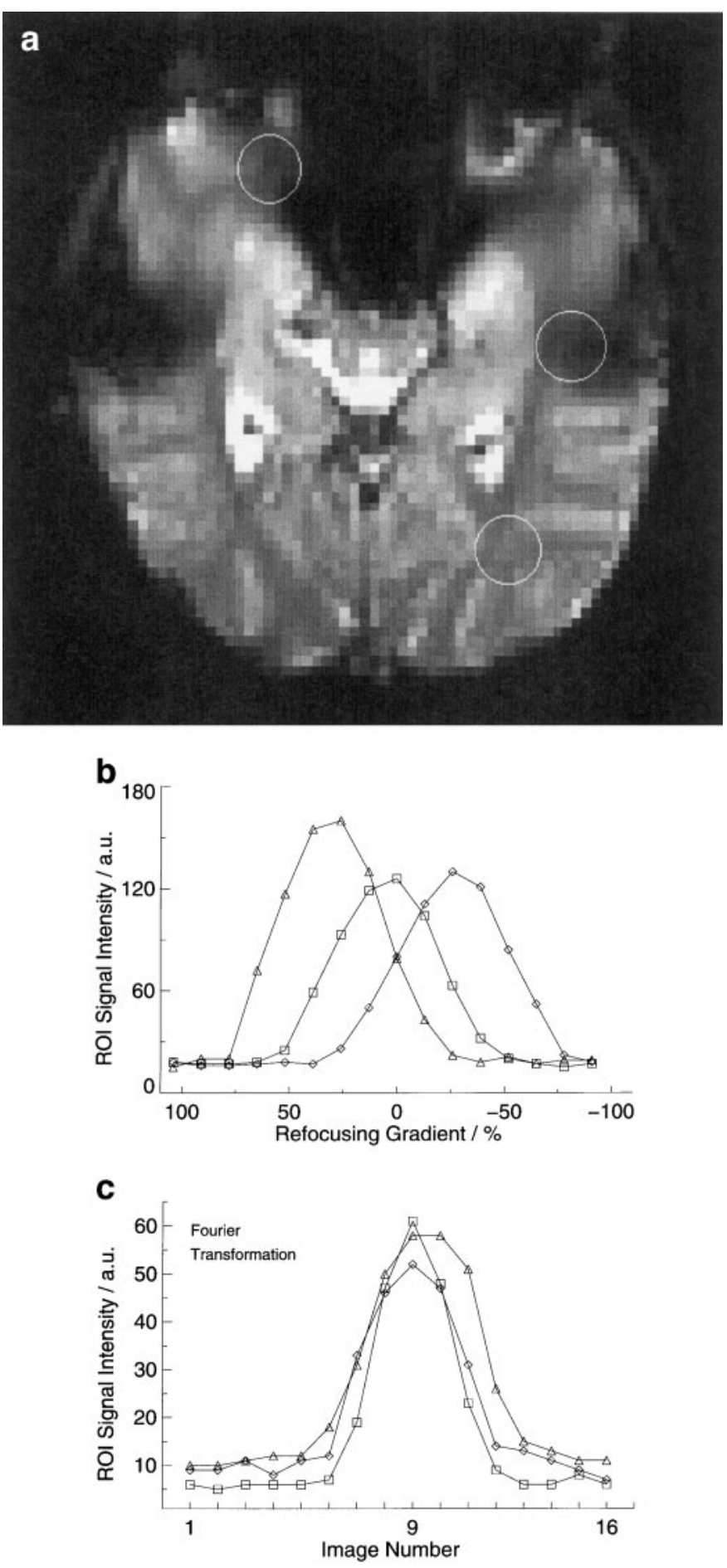

FIG. 1. Inhomogeneity-induced signal losses in the brain of a single subject. (a) EPI $\left(2.0 \times 2.0 \mathrm{~mm}^{2}\right.$ resolution, $4 \mathrm{~mm}$ thickness, TR/TE $=$ $2000 / 53 \mathrm{~ms}$, flip angle $70^{\circ}$ ) of a transverse-to-coronal section along the orientation of the hippocampus. (b) Region-of-interest (ROI) signal intensities of the inferior frontal region (triangles), occipital cortex (squares), and inferior temporal cortex (diamonds) as a function of the refocusing gradient strength (gradient integral ranging from 9.6 to $-8.4 \mathrm{mT} \mathrm{m}^{-1} \mathrm{~ms}$ ). The ROIs are indicated in (a). (c) Corresponding ROI signal intensities after Fourier transformation of 16 acquisitions with different refocusing gradients as a function of image number. 

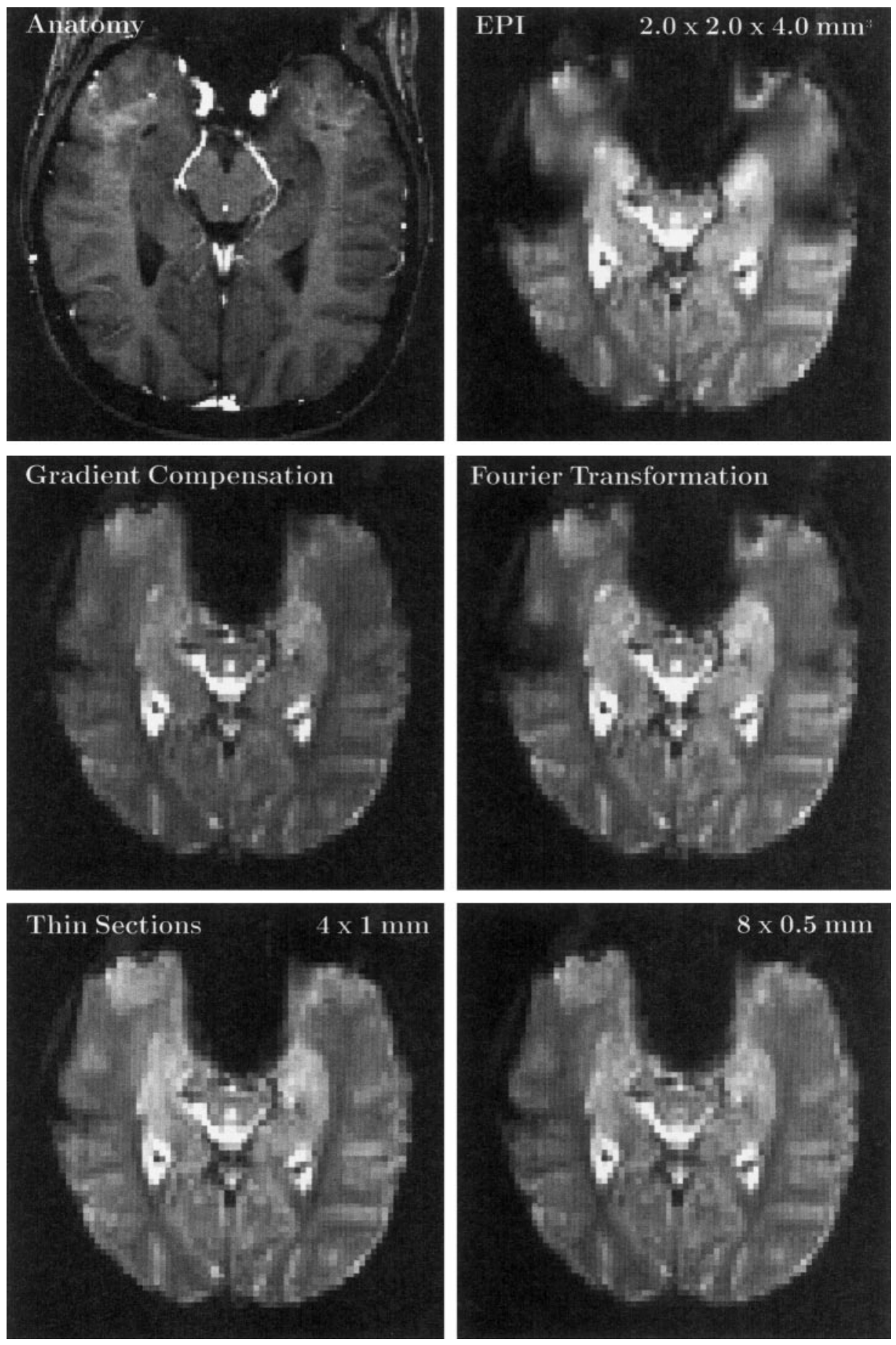

FIG. 2. Inhomogeneity-induced signal losses and corrections in a section covering the hippocampus of the same subject as in Fig. 1. (Top left) T1-weighted anatomy (RF-spoiled FLASH, TR/TE $=70 / 6 \mathrm{~ms}$, flip angle $60^{\circ}$ ); (top right) EPI (parameters as in Fig. 1a); (middle left) gradient-compensated MIP map $\left(\mathrm{TR} / \mathrm{TE}=125 / 53 \mathrm{~ms}\right.$, flip angle $\left.15^{\circ}\right)$; (middle right) gradient-compensated FT map; (bottom left) sum of four 1-mm multislice images; and (bottom right) sum of eight 0.5 -mm multislice images $\left(\mathrm{TR} / \mathrm{TE}=2000 / 54 \mathrm{~ms}\right.$, flip angle $\left.70^{\circ}\right)$.

reveals that a residual borderzone of the frontal lobe, the anterior hippocampus and amygdala, and the midbrain with the nasal cavity is not compensated for by either of the two correction strategies. This finding may be attributed to the presence of very strong and differently oriented susceptibility gradients in these regions that not only dephase excited spins in all three gradient axes but also distort the slice profile in such a way that parts of the target section are not even excited. 

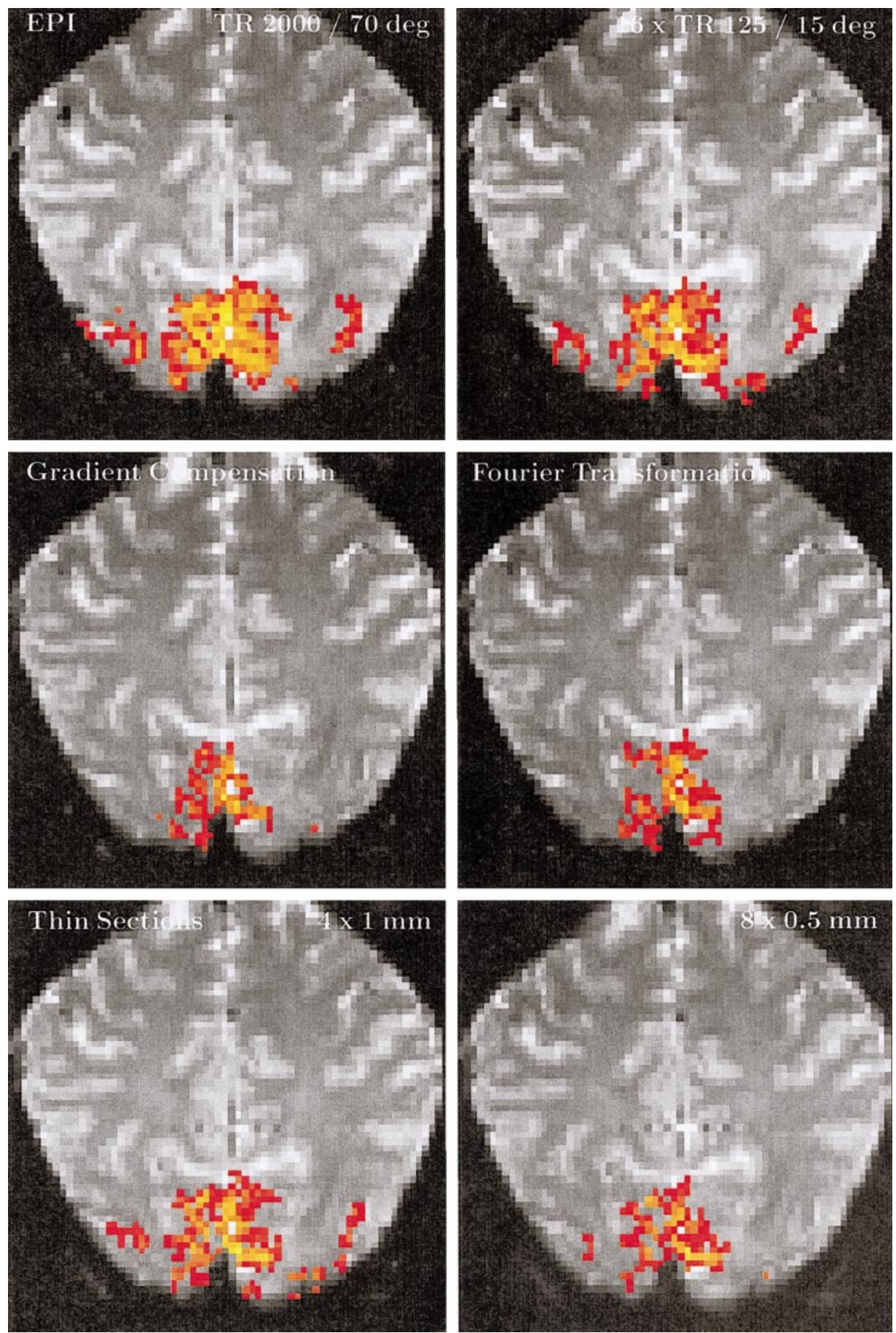

FIG. 3. Activation maps of the visual cortex of a single subject acquired with and without inhomogeneity correction. The maps correspond to acquisitions based on (top left) EPI (parameters as in Fig. 1a); (top right) the normalized sum of sixteen 4-mm images (TR/TE $=125 / 53 \mathrm{~ms}$, flip angle $15^{\circ}$ ) using the same refocusing gradient strength; (middle left) gradient-compensated MIP images (TR/TE $=125 / 53 \mathrm{~ms}$, flip angle $15^{\circ}$ ); (middle right) gradient-compensated FT images; (bottom left) the sum of four 1-mm multislice images; and (bottom right) the sum of eight 0.5 -mm multislice images (TR/TE $=2000 / 54$ ms, flip angle $70^{\circ}$ ).

\section{Functional Mapping of the Visual System}

A comparison of visual activation maps obtained with and without inhomogeneity corrections are shown in Figs. 3 and 4 for two different subjects. In a first step we investigated the influence of replacing a conventional EPI acquisition (top left) by the normalized sum of a sequential acquisition of 16 images (top right). As summarized in Table 1 the mean number of 

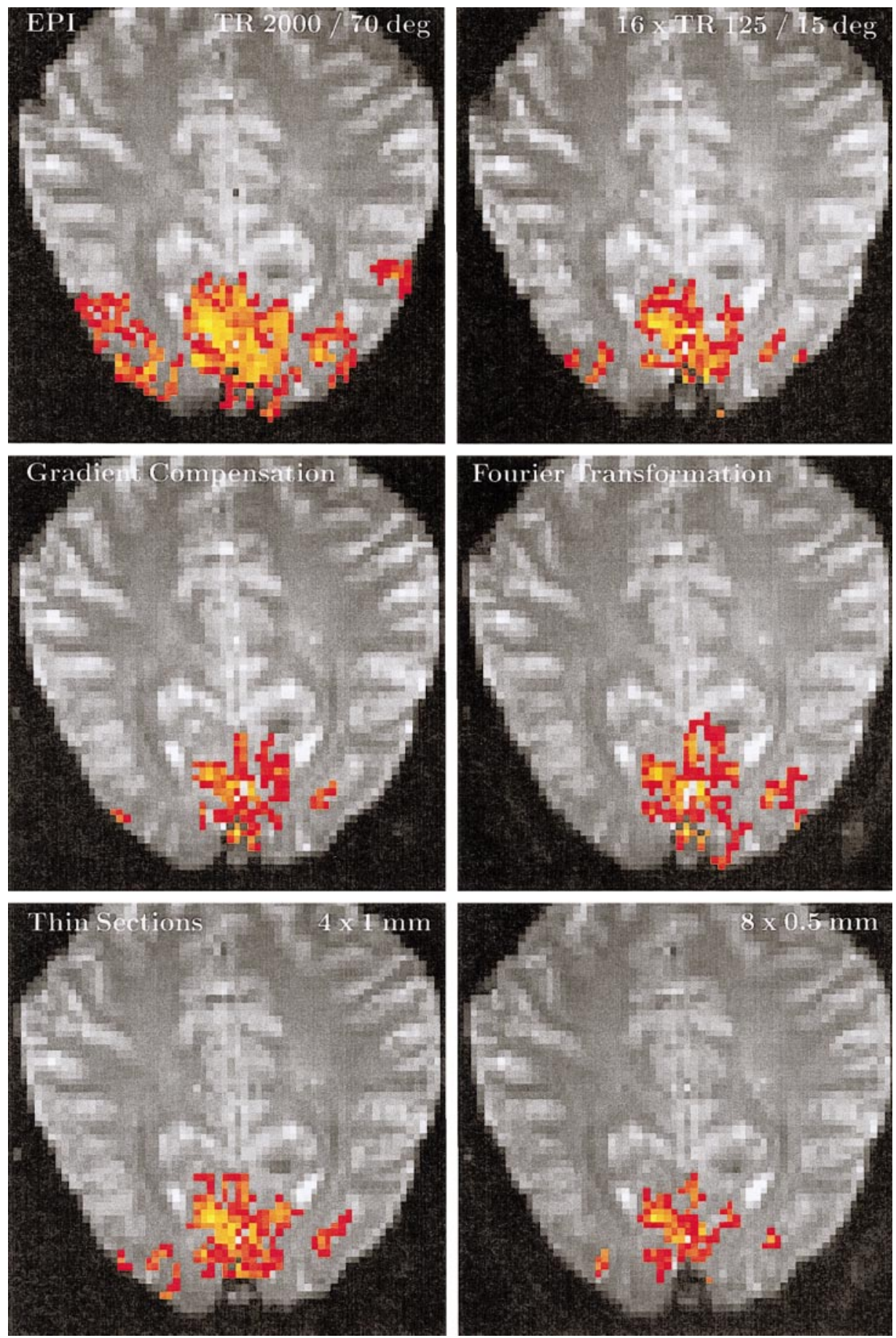

FIG. 4. Activation maps of the visual cortex as in Fig. 3 but for a different subject (all parameters as in Fig. 3).

activated pixels in the respective maps decreased by about $27 \%$. This is somewhat larger than the theoretically expected reduction of the SNR calculated from the respective steadystate signal intensities. It probably reflects the additive adverse effects of reduced SNR onto the statistical treatment of stimulus-correlated signal alterations. Figures 3 and 4 also demonstrate a marked intersubject variability, with one subject show- ing almost no difference between the two maps and the other subject exhibiting a pronounced reduction of the activated area for the 16-image procedure.

The TR (2000 vs $125 \mathrm{~ms})$ and flip angle $\left(70^{\circ}\right.$ vs $\left.15^{\circ}\right)$ adjustments were necessary to retain similar image contrasts when moving toward sequential acquisitions of gradient-compensated images. The middle parts of Figs. 3 and 4 show the 
TABLE 1

N umber of Activated Pixels in the Visual Cortex (Mean \pm SD, $n=6$ ) for a 4-mm E PI Section at $2.0 \times 2.0 \mathrm{~mm}^{2}$ Resolution with and without Inhomogeneity Correction

\begin{tabular}{lll}
\hline \multicolumn{1}{c}{ Method } & \multicolumn{1}{c}{ Variant } & Activated pixels \\
\hline \multirow{2}{*}{ Standard } & TR 2000/70 & $344 \pm 96$ \\
& $16 \times$ TR $125 / 15^{\circ}$ & $252 \pm 82$ \\
Compensation & Maximum intensity projection & $148 \pm 44$ \\
& Fourier transformation & $173 \pm 41$ \\
Thin sections & $4 \times 1.0 \mathrm{~mm}$ & $228 \pm 47$ \\
& $8 \times 0.5 \mathrm{~mm}$ & $133 \pm 45$ \\
\hline
\end{tabular}

${ }^{a} n=4$.

results obtained for the gradient compensation method either directly as a MIP map (left) or after FT (right). Both variants yield less activation than with constant refocusing. The slightly better FT maps exhibit a 50\% reduction of activated pixels. This is mainly because only 3 of the 16 acquired images with sufficient SNR contribute to the final map.

Relative to gradient compensation the simple use of contiguous $1-\mathrm{mm}$ thin sections and their subsequent summation resulted in significantly improved activation maps leading to only a 33\% reduction of activated pixels (bottom left). Intuitively, susceptibility-induced artifacts should be further reduced in even thinner sections. However, such acquisitions do not translate into better activation maps but cause a significant reduction of activated pixels (bottom right). This observation reflects the fact that a subdivision of the original plane into $N$ thin sections decreases the SNR of the summed image according to $1 / \sqrt{N}$ (i.e., $1 / N \times \sqrt{N}$ ). Because a lower SNR most likely decreases the number of admitted pixels in the statistical analysis, the activation maps derived from the 1-mm sections are even better than expected for a 50\% reduction of the SNR. This promising result seems to reflect the elimination of artifactual signal fluctuations in the 1-mm images as opposed to 4-mm acquisitions. In fact, a more consistent activation across subjects with significantly reduced intersubject variability is also documented in Table 1 which shows that maps derived from 1-mm sections retain $\frac{2}{3}$ of the original number of activated pixels with only half of the standard deviation. In practice, one has to find a compromise between thick sections with sufficient SNR for a reliable analysis of functional responses and thin sections that effectively avoid susceptibility-induced dephasing. For the present experimental conditions, the optimum thickness seems to be $1 \mathrm{~mm}$.

\section{CONCLUSION}

We investigated two different methods for correcting inhomogeneity artifacts in gradient-echo EPI of the human brain at $2.0 \times 2.0 \times 4.0 \mathrm{~mm}^{3}$ resolution. The performance of the strategies was judged by their ability to recover signal losses in
$\mathrm{T} 2 *$-weighted images of the base of the brain and to map functional activation of the visual cortex, respectively. In comparison to standard 4-mm EPI acquisitions both the gradient compensation and the thin section method were capable of partly alleviating the inhomogeneity problem in the hippocampal formation excluding anterior regions. However, both methods partially compromised functional mapping in the visual cortex due to reduced SNR.

In general, compensation of the slice-selection gradient was found to be less effective than the use of thin sections. Multislice EPI of contiguous thin slices is simple as only minimal technical adjustments are required. Because 1-mm sections seem to be sufficient for imaging the hippocampal formation, a temporal resolution of $2.0 \mathrm{~s}$ is not restricted to the acquisition of a single 4-mm section but allows the EPI coverage of approximately a $20-\mathrm{mm}$ volume. In fact, suitable subsets of sections with arbitrary thicknesses may be selected in retrospect. Pertinent studies of hippocampal activation are in progress.

\section{REFERENCES}

1. I. R. Young, I. J . Cox, D. J . Bryant, and G. M. Bydder, The benefits of increasing spatial resolution as a means of reducing artifacts due to field inhomogeneities, Magn. Reson. Imaging 6, 585-590 (1988).

2. E. M. Haacke, J. A. Tkach, and T. B. Parrish, Reduction of $T_{2}^{*}$ dephasing in gradient field-echo imaging, Radiology 170, 457- 462 (1989).

3. J. Frahm, K. D. Merboldt, and W. Hänicke, Functional MRI of human brain activation at high spatial resolution, Magn. Reson. Med. 29, 139-144 (1993).

4. A. J esmanowicz, B. B. Biswal, and J . S. Hyde, Reduction in GR-EPI intravoxel dephasing using thin slices and short TE, in Proc. Int. Soc. Magn. Reson. Med., 7th Annual Meeting, Philadelphia, p. 1619 (1999).

5. Z. H. Cho and Y. M. Ro, Reduction of susceptibility artifact in gradient-echo imaging, Magn. Reson. Med. 23, 193-200 (1992).

6. N. K. Chen and A. Wyrwicz, Removal of intravoxel dephasing artifact in gradient-echo images using a field-map based RF refocusing technique. Magn. Reson. Med. 42, 807-812 (1999).

7. G. H. Glover and S. Lai, Reduction of susceptibility effects in BOLD $\mathrm{fMRI}$ using tailored RF pulses, in Proc. Int. Soc. Magn. Reson. Med., 6th Annual Meeting, Sydney, p. 298 (1998).

8. J. Frahm, K. D. Merboldt, and W. Hänicke, Direct FLASH MR imaging of magnetic field inhomogeneities by gradient compensation, Magn. Reson. Med. 6, $474-480$ (1988).

9. J. Frahm, K. D. Merboldt, and W. Hänicke, The influence of the slice-selection gradient on functional MRI of human brain activation, J. Magn. Reson. B 103, 91-93 (1994).

10. R. J . Ordidge, J. M. Gorell, J. C. Deniau, R. A. Knight, and J. A. Helpern, Assessment of relative brain iron concentrations using $\mathrm{T}_{2}$-weighted and $\mathrm{T}_{2}^{*}$-weighted MRI at 3 Tesla, Magn. Reson. Med. 32, 335-341 (1994).

11. J . Frahm, K. D. Merboldt, and W. Hänicke, The effects of intravoxel dephasing and incomplete slice refocusing on susceptibility contrast in gradient-echo MRI, J. Magn. Reson. B 109, 234-237 (1995).

12. R. T. Constable, Functional MR imaging using gradient-echo echo- 
planar imaging in the presence of large static field inhomogeneities, J. Magn. Reson. Imaging 5, 746-752 (1995).

13. Q. X. Yang, G. D. Williams, R. J . Demeure, T. J . Mosher, and M. B. Smith, Removal of local field gradient artifacts in $\mathrm{T}_{2}^{*}$-weighted images at high fields by gradient-echo slice excitation profile imaging, Magn. Reson. Med. 39, 402-409 (1998).

14. Q. X. Yang, W. B. Edmister, K. K. Kwong, R. J. Demeure, and M. B. Smith, Removal of the signal loss artifacts in $\mathrm{T}_{2}^{*}$-weighted EPI, in Proc. Int. Soc. Magn. Reson. Med., 6th Annual Meeting, Sydney, p. 1443 (1998)

15. D. Cordes, B. J . Mock, P. A. Turski, and J . A. Sorenson, Functional magnetic resonance imaging using optimized z-gradient refocusing, in Proc. Int. Soc. Magn. Reson. Med., 6th Annual Meeting, Sydney, p. 1446 (1998).

16. G. H. Glover, 3D Z-shim method for reduction of susceptibility effects in BOLD fMRI, Magn. Reson. Med. 42, 290-299 (1999).

17. V. A. Stenger, F. E. Boada, and D. C. Noll, Gradient compensation method for the reduction of susceptibility artifacts for spiral fMRI data acquisition, in Proc. Int. Soc. Magn. Reson. Med., 7th Annual Meeting, Philadelphia, p. 538 (1999).

18. Q. X. Yang, R. J . Demeure, and M. B. Smith, Rapid whole brain $\mathrm{T}_{2}^{*}$ echo planar imaging with removal of susceptibility artifacts, in Proc. Int. Soc. Magn. Reson. Med., 7th Annual Meeting, Philadelphia, p. 537 (1999).

19. G. Krüger, P. Fransson, K. D. Merboldt, and J . Frahm, Does stimulus quality affect the physiologic MRI responses to brief visual activation? Neuroreport 10, 1277-1281 (1999).

20. P. A. Bandettini, A. Jesmanowicz, E. C. Wong, and J. H. Hyde, Processing strategies for time-course data sets in functional MRI responses to visual activation in humans, Magn. Reson. Med. 30, 161-173 (1993).

21. A. Kleinschmidt, M. Requardt, K. D. Merboldt, and J . Frahm, On the use of temporal correlation coefficients for magnetic resonance mapping of functional brain activation. Individualized thresholds and spatial response delineation, Int. J . Imaging System Technol. 6, 238-244 (1995). 